\title{
Editorial
}

Nephrology

\section{Cardiovascular Benefits of a Diet Enriched in Fruits and Vegetables}

\author{
Biff F. Palmer ${ }^{a}$ Deborah J. Clegg ${ }^{b, c}$ \\ a Professor of Internal Medicine, Department of Internal Medicine, University of Texas Southwestern Medical Center, \\ Dallas, TX, USA; ${ }^{b}$ Distinguished Scientist-in-Residence, Department of Health Studies, College of Arts and Sciences, \\ American University, Washington, DC, USA; ${ }^{\circ}$ Visiting Professor, Biomedical Sciences, Diabetes and Obesity Research \\ Institute, Cedars-Sinai Medical Center, Los Angeles, CA, USA
}

The kidney plays a critical role in the regulation of acid-base balance by reclaiming filtered bicarbonate and regenerating an additional amount of bicarbonate to replenish body buffers consumed by net endogenous acid production. The elimination of the acid load is accomplished by urinary excretion of protons primarily as ammonium and to a lesser extent by titratable acidity. Daily net acid production is approximately $1 \mathrm{mEq}$ of $\mathrm{H}^{+}$per kilogram body weight and represents the sum of protons derived from the metabolism of ingested protein minus the difference between bicarbonate derived from metabolism of organic acid anions (originating predominately from ingested fruits and vegetables) and organic acid anions lost in urine. As a result, the net endogenous acid production is increased with diets high in protein intake and reduced with low protein intake or diets enriched in fruits and vegetables.

With the development of chronic kidney disease (CKD), acid excretion is maintained by an adaptive increase in ammonium excretion per nephron. Once the glomerular filtration rate falls to values corresponding to

\section{KARGER}

(๑) 2019 S. Karger AG, Basel

E-Mail karger@karger.com

www.karger.com/ajn
CKD stage 3 or 4 , the reduction in the total number of functioning nephrons limits the total ammonium excretion predisposing to the development of overt metabolic acidosis. Metabolic acidosis has been linked to a number of adverse effects to include muscle protein degradation and negative nitrogen balance, bone disease, stimulation of inflammation, impaired insulin secretion, progression of $\mathrm{CKD}$, and increased mortality. For these reasons, the preservation of acid/base balance is critical in CKD management and justify clinical practice guidelines recommending the administration of base when the serum bicarbonate concentration is $<22 \mathrm{mEq} / \mathrm{L}$.

Alkali therapy is typically given as sodium bicarbonate in a dose of $0.5-1 \mathrm{mEq} / \mathrm{kg} /$ day. While effective, this approach is associated with increased sodium intake and can exacerbate the volume expansion and hypertension commonly present in CKD. In addition, some patients are intolerant due to complications of bloating. Since the modern western diet gives rise to increased net acid production, an alternative approach is to increase the consumption of fruits and vegetables, which is associated 
with alkali precursors and is not accompanied by a salt load. This approach was tested by Goraya et al. [1], in 108 macroalbuminuric, non-diabetic patients with stage 3 $\mathrm{CKD}$ and plasma $\mathrm{TCO}_{2} 22-24 \mathrm{mEq} / \mathrm{L}$ randomized to usual care or interventions designed to reduce dietary acid by $50 \%$ using sodium bicarbonate or base-producing fruits and vegetables. Fruits and vegetables were provided to the family as well as to the participants and included apples, apricots, oranges, peaches, pears, raisins, and strawberries. Vegetables provided were carrots, cauliflower, eggplant, lettuce, potatoes, spinach, tomatoes, and zucchini. After 3 years of follow-up, dietary acid reduction with sodium bicarbonate or base-producing fruits and vegetables reduced urine excretion of angiotensinogen, an index of kidney angiotensin II levels, and preserved eGFR to a greater extent than usual care.

The study also demonstrated that dietary acid reduction is protective in CKD even when the plasma $\mathrm{TCO}_{2}$ $>22 \mathrm{mEq} / \mathrm{L}$, a level for which guidelines do not recommend treatment. In this regard, studies in rodent models and humans indicate that reductions in plasma bicarbonate are preceded by a subclinical metabolic acidosis where intracellular and bone buffers mask the early retention of hydrogen ions. At this stage, buffer stores are already reduced even though plasma bicarbonate concentration and $\mathrm{pH}$ may be normal.

In this issue, the authors describe the 5 year effect of the 3 interventions on eGFR as the primary analysis but also report on indicators of cardiovascular risk as a secondary analysis [2]. Similar to the original report, at 5 years, patients treated with fruits and vegetables and bicarbonate had higher plasma $\mathrm{TCO}_{2}$ and lesser decline in kidney function when compared to patients under usual care. Despite comparable improvements in metabolic acidosis and kidney function, the group treated with fruits and vegetables had better improvement in biomarkers of cardiovascular risk to include systolic blood pressure, body mass index, lipid parameters to include LDL, Lp(a), and higher serum vitamin K1 (low values associated with coronary calcification) when compared to those under bicarbonate therapy or usual care. These additional findings lend support to the importance of consumption of foods to alter acid-base balance above and beyond pharmacology.

Diets enriched in fruit and vegetables are well known to be associated with a reduction in blood pressure [3]. In addition to less sodium content, these diets are enriched in potassium. Epidemiologic studies have established that $\mathrm{K}^{+}$intake is inversely related to the prevalence of hypertension. In addition, $\mathrm{K}^{+}$supplements and avoidance of hypokalemia lower blood pressure in hypertensive subjects, whereas blood pressure increases in hypertensive subjects placed on a low $\mathrm{K}^{+}$diet. In addition to effects on sympathetic nerve activity and vascular effects, high $\mathrm{K}^{+}$ intake is natriuretic by exerting an inhibitory effect on the thiazide sensitive cotransporter in the initial portion of the distal convoluted tubule [4]. This natriuretic effect is more pronounced in individuals with high salt intake and in trials where black individuals were a majority of the participants.

Diets enriched in fruits and vegetables are also high in fiber and this has consistently been associated with favorable outcomes related to body weight regulation and improvement in lipids [5]. High fiber increases intraluminal viscosity in the intestine leading to reduced glucose and free fatty acid absorption, thereby promoting fat oxidation and reduction in fat storage. Reduced glucose absorption associated with the fiber intake decreases insulin secretion, thereby minimizing the risk of reactive hypoglycemia and augmentation of hunger that often occurs following high carbohydrate meals during the post-absorption period. Dietary fiber may further suppress hunger through stimulatory effects on gut hormones that influence satiety to include cholecystokinin and glucagonlike-peptide-1. Plant-sourced foods are also the primary source of vitamin $\mathrm{K} 1$ accounting for the higher levels noted in the study.

A diet enriched in fruits and vegetables also provides a means to maintain adequate protein intake with less tendency to cause phosphorus overload. Inorganic phosphate is used in excess as a preservative and flavor enhancer in processed foods and it is estimated that nearly a quarter of adults consume phosphate at several fold higher levels than the recommended daily allowance. High phosphate intake in normal rodents is associated with pathologic remodeling in cardiac muscle and unfavorable effects on exercise tolerance [6]. Phosphorus in animal protein is in the form of organic phosphate, which is readily hydrolyzed and absorbed. Organic phosphorus from plant protein is less well absorbed when compared to phosphorus from animal proteins because phosphorus from plants is in the form of phytates and mammals lack phytases, which are required to break down the phytates. Despite an equivalent amount of protein and phosphorus concentrations in the diet, ingestion of a plant-based diet lowers serum phosphorus levels and significantly reduces FGF23 levels consistent with reduced gastrointestinal absorption of phosphate.

At this time, one can safely say that a diet enriched in fruits and vegetables provides a comparable benefit to so- 
dium bicarbonate by improving metabolic acidosis and eGFR preservation. The study of Goraya et al. [2] suggests that fruits and vegetables may be the preferred modality, given the improvement in cardiovascular risk indicators, which is above and beyond what was achieved with the intake of $\mathrm{HCO} 3$ tablets and is in line with the findings of other recent studies demonstrating that this type of diet slows the progression of CKD and reduced mortality in adults undergoing maintenance hemodialysis $[7,8]$.

The long-standing practice of limiting plant-based foods to avoid hyperkalemia in those with CKD should not be a blanket recommendation but rather individualized. Hyperkalemia was not found in patients with stage $4 \mathrm{CKD}$ given a diet containing $70 \%$ protein from plants.
In fact, eating a diet enriched in fruits and vegetables shows little to no correlation with the measurement of the predialysis potassium concentration in dialysis patients. The alkaline load in $\mathrm{K}^{+}$-rich foods may actually facilitate kidney excretion, since alkali present in such a diet directly affects the determinants of $\mathrm{K}^{+}$transport in the DCT. With more advanced CKD or end-stage-kidney disease excretion of $\mathrm{K}^{+}$in the stool may be enhanced due to increased stool bulk from dietary fiber in the plantbased diet. The administration of new $\mathrm{K}^{+}$binding drugs may prove useful to allow liberalization of the diet to allow for the metabolic benefits outlined above in patients at risk for hyperkalemia.

\section{References}

1 Goraya N, Simoni J, Jo CH, Wesson DE. Treatment of metabolicacidosisin patients with stage 3 chronic kidney disease with fruits and vegetables or oral bicarbonate reduces urine angiotensinogen and preserves glomerular filtration rate. Kidney Int. 2014 Nov;86(5):1031-8.

2 Goraya N, Munoz-Maldonado Y, Simoni J, Wesson DE. Fruit and vegetable treatment of Chronic Kidney Disease-related metabolic acidosis reduces cardiovascular risk better than Sodium Bicarbonate. Am J Nephrol. 2019;49:438-48.
3 Palmer BF, Clegg DJ. Achieving the benefits of a high-potassium, Paleolithic diet, without the toxicity. Mayo Clin Proc. 2016 Apr;91(4): 496-508.

4 Palmer BF. Regulation of Potassium Homeostasis. Clin J Am Soc Nephrol. 2015 Jun;10(6): 1050-60.

5 Bozzetto L, Costabile G, Della Pepa G, Ciciola P, Vetrani C, Vitale M, et al. Dietary Fibre as a Unifying Remedy for the Whole Spectrum of Obesity-Associated Cardiovascular Risk. Nutrients. 2018 Jul;10(7):E943.

6 Peri-Okonny P, Baskin KK, Iwamoto G, Mitchell JH, Smith SA, Kim HK, et al. HighPhosphate Diet Induces Exercise Intolerance and Impairs Fatty Acid Metabolism in Mice. Circulation. 2019 Mar;139(11):1422-34.
7 Kim HW, Park JT, Yoo TH, Lee J, Chung W, Lee KB, et al.; KNOW-CKD Study Investigators. Urinary Potassium Excretion and Progression of CKD. Clin J Am Soc Nephrol. 2019 Mar; 14(3):330-40

8 Saglimbene V, Wong G, Ruospo M, Palmer S, Garcia-Larsen V, Natale P, et al. Fruit and Vegetable Intake and Mortality in Adults undergoing Maintenance Hemodialysis. CJASN. 2019 Feb;14(2):250-60. 